ARTICLE

\title{
Joint mouse-human phenome-wide association to test gene function and disease risk
}

\author{
Xusheng Wang ${ }^{1,2, \star}$, Ashutosh K. Pandey ${ }^{1, \star}$, Megan K. Mulligan ${ }^{1}$, Evan G. Williams ${ }^{3}$, Khyobeni Mozhui ${ }^{1}$,
} Zhengsheng $\mathrm{Li}^{1}$, Virginija Jovaisaite ${ }^{3}$, L. Darryl Quarles ${ }^{4}$, Zhousheng Xiao ${ }^{4}$, Jinsong Huang ${ }^{1,4}$, John A. Capra ${ }^{5}$, Zugen Chen ${ }^{6}$, William L. Taylor ${ }^{7}$, Lisa Bastarache ${ }^{5}$, Xinnan Niu ${ }^{5}$, Katherine S. Pollard ${ }^{8,9}$, Daniel C. Ciobanu ${ }^{1,10}$,

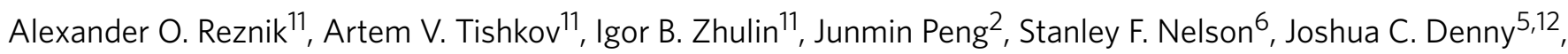
Johan Auwerx ${ }^{3}$, Lu Lu$^{1} \&$ Robert W. Williams ${ }^{1}$

Phenome-wide association is a novel reverse genetic strategy to analyze genome-tophenome relations in human clinical cohorts. Here we test this approach using a large murine population segregating for $\sim 5$ million sequence variants, and we compare our results to those extracted from a matched analysis of gene variants in a large human cohort. For the mouse cohort, we amassed a deep and broad open-access phenome consisting of $\sim$ 4,500 metabolic, physiological, pharmacological and behavioural traits, and more than 90 independent expression quantitative trait locus (QTL), transcriptome, proteome, metagenome and metabolome data sets-by far the largest coherent phenome for any experimental cohort (www.genenetwork.org). We tested downstream effects of subsets of variants and discovered several novel associations, including a missense mutation in fumarate hydratase that controls variation in the mitochondrial unfolded protein response in both mouse and Caenorhabditis elegans, and missense mutations in Col6a5 that underlies variation in bone mineral density in both mouse and human.

\footnotetext{
${ }^{1}$ Department of Genetics, Genomics and Informatics, University of Tennessee Health Science Center, Memphis, Tennessee 38163, USA. ${ }^{2}$ St Jude Proteomics Facility, St Jude Children's Research Hospital, Memphis, Tennessee 38105, USA. ${ }^{3}$ Laboratory of Integrative and Systems Physiology, School of Life Sciences, École Polytechnique Fédérale de Lausanne, Lausanne 1015, Switzerland. ${ }^{4}$ Department of Medicine, University of Tennessee Health Science Center, Memphis, Tennessee 38163, USA. ${ }^{5}$ Department of Biomedical Informatics, Vanderbilt University School of Medicine, Nashville, Tennessee 37232, USA. ${ }^{6}$ Department of Human Genetics, University of California, Los Angeles, California 90095, USA. ${ }^{7}$ Molecular Resource Center, University of Tennessee Health Science Center, Memphis, Tennessee 38163, USA. ${ }^{8}$ Gladstone Institutes, San Francisco, California 94158, USA. ${ }^{9}$ Division of Biostatistics and Institute for Human Genetics, University of California, San Francisco, California 94158, USA. ${ }^{10}$ Animal Science Department, University of Nebraska, Lincoln, Nebraska 68583, USA.

${ }^{11}$ Joint Institute for Computational Sciences, University of Tennessee-Oak Ridge National Laboratory, Oak Ridge, Tennessee 37831, USA. ${ }^{12}$ Department of Medicine, Vanderbilt University School of Medicine, Nashville, Tennessee 37232, USA. * These authors contributed equally to this work. Correspondence and requests for materials should be addressed to R.W.W. (email: rwilliams@uthsc.edu).
} 
dentifying sequence variants that control or modify sets of linked phenotypes is fundamental to understanding the molecular basis of both Mendelian and complex traits ${ }^{1-4}$. A variety of reverse genetic approaches to induce loss- and gain-of-function have been used to causally tie DNA variants to discrete phenotypes ${ }^{5}$. However, reverse genetics presents two challenges. The first is evaluating a potentially broad spectrum of phenotypes, biomarkers and endophenotypes that are downstream of sequence variants at different stages of development, in different tissues and cells, and under different conditions. The second is evaluating the impact of these variants across different genetic backgrounds that influence trait penetrance. Phenome-wide association studies (PheWAS) address both challenges ${ }^{6,7}$.

To establish the first murine resource for phenome-wide association, we have used a large cohort of recombinant inbred strains-the BXD family-that were generated by crossing two fully inbred parental strains-C57BL/6J (B6) and DBA/2J (D2). This family consists of $\sim 150$ isogenic lines, and as we show here, this family segregates for over five million common variants and $\sim 12,000$ missense mutations. To accompany these genomic data, we assembled a high-coverage phenome with over 4,500 quantitative metabolic, physiological, pharmacological, toxicological, morphometric and behavioural phenotypes, along with linked references to the primary literature. This BXD phenome also includes $\sim 90$ large open-access expression quantitative trait locus (eQTL) studies generated over the past decade as well as recent metagenomic, metabolomics and proteomic components ${ }^{8-10}$. Roughly half of the eQTL data sets are experimental, developmental or related to aging. Almost all phenome data types and genome sequence are accessible online as a companion to this paper.

The impact of $\sim 12,000$ potentially damaging sequence variants was evaluated by systematically scanning them against the phenome at molecular, cellular and behavioural levels. We discovered new associations, some of which were subsequently validated in a large human clinical cohort ${ }^{6,7}$. For example, missense mutations in Col6a5 were linked to variation in bone mineral density in both mouse and human. However, downstream effects of allelic variants with presumed deleterious effects on gene expression or protein structure are often small or undetectable. This may often be due to a lack of technical sensitivity and power, or due to molecular and developmental compensation.

\section{Results}

Phenomes. Phenome data were generated using a large cohort of recombinant inbred strains-the BXD family-that was derived by crossing two fully inbred parents-C57BL/6J (B6) and DBA/2J (D2). Members of the BXD family collectively segregate for all sequence variants that distinguish the two parents-and in this cross these are by definition common variants. There are also interesting rare but still undefined alleles unique to each family member. The level of both genetic and phenotypic variation between parents and among the strains is high (Fig. 1a). This BXD phenome includes $\sim 4,500$ quantitative metabolic, physiological, pharmacological, toxicological, morphometric and behavioural phenotypes (Fig. 1b). These traits are almost all quantitative and have been systematically grouped into 15 major phenotype categories (Supplementary Data 1). We have also generated and assembled a large molecular phenome that includes expression phenotypes from $\sim 90$ large open-access eQTL studies generated over the past decade (Fig. $1 \mathrm{~b}$ and Supplementary Data 2). On average $1.5 \times 10^{6}$ mRNA, $1.7 \times 10^{4}$ proteomic and $6.8 \times 10^{3}$ metabolomic assays are available per strain (Fig. 1b). Most phenotypes vary markedly across strains within the family. For example, effects of high-fat and low-fat diets on adult body weight vary substantially across genotypes (Fig. 1c). Similarly mRNA and protein expression of, for example, $B c k d h b$ and many other mRNA, proteins, and metabolites vary greatly (Fig. 1d) ${ }^{10}$. The online availability of well-organized classic and molecular traits from the BXD family (see www.genenetwork.org) provides the foundation for multiscalar phenome scans of any putatively functional sequence variant.

The human phenome used in this study is a large electronic health record (EHR-linked cohort, BioVU https://victr.vanderbilt.edu/pub/biovu/). BioVU currently contains $>190,000$ DNA samples linked to de-identified medical records to provide a large, clinically relevant human resource to study genotype-phenotype associations; 29,722 of these individuals have extant exome variant data, which was used for matched mouse-to-human PheWAS in this study.

Whole-genome sequencing and variant detection. To obtain accurate information on sequence variants across the BXD family (Fig. 2a), we generated $\sim 8.26$ billion reads using six paired-end libraries with different insert sizes from the D2 parent using two sequencing platforms. A total of 4.5 billion reads $(262 \mathrm{~Gb}$ nucleotides) were aligned to the genome of the other parent of the BXD family (B6) that serves as the mouse reference genome. The mouse genome consists of $\sim 2.6 \mathrm{~Gb}$, and we generated $\sim 100$-fold coverage (Fig. 2b and Supplementary Data 3) and sequenced $99.96 \%$ of the reference genome excluding gaps and regions of low complexity.

The parents of the BXD cohort differ at 4.8 million singlenucleotide polymorphisms (SNPs) (Fig. 2c) at a high-confidence threshold, including 4,160,570 extracted from the SOLiD platform and 4,090,000 SNPs from Illumina (Supplementary Fig. 1a). Recently, Keane et al. ${ }^{4}$ generated $\sim 25$-fold coverage of the D2 genome using the Illumina platform. We found a reasonably high concordance $(94.4 \%)$ in SNP detection between Illumina data and that of Keane et al. (Supplementary Fig. 1b). The distribution of SNPs across functionally distinct genomic regions is provided in Supplementary Fig. 1c and Supplementary Note 1 . We resequenced a subset of 262 platform-specific SNPs. False positive rates (FPR) were $2.34 \%$ and $3.73 \%$ for SOLiD and Illumina platforms, respectively. Assuming that all 3,375,198 SNPs detected by both systems are valid, the FPR are $0.44 \%$ for SOLiD and $0.65 \%$ for Illumina.

We defined 35,068 coding SNPs (cSNPs), of which 23,089 are silent (synonymous) and 11,979 are missense (non-synonymous) (Supplementary Data 4). Approximately $16 \%$ and $4 \%$ of non-synonymous SNPs have potentially deleterious effects on protein function as assessed using Polymorphism Phenotyping 2 (PolyPhen 2) (ref. 11) and Sorting Intolerant From Tolerant (SIFT) (ref. 12), respectively (Supplementary Data 4). Approximately 2\% (210 SNPs) were defined as deleterious by both algorithms (Fig. 2d and Supplementary Data 4). We identified 58 nonsense SNPs in 53 genes, including 42 stop codon gains and 16 stop codon losses in D2 (Supplementary Data 5). The functional consequence of 210 deleterious missense variants and 58 nonsense variants were further evaluated by comparative genomic analysis (Supplementary Data 6 and 7 and Supplementary Note 1). In addition, 79 missense variants were confirmed by mass spectrometry-based proteomics (Supplementary Data 8 and Supplementary Note 1).

Most SNPs (98\%) occur within noncoding regions (noncoding SNPs, ncSNPs). An impact score of each ncSNP was calculated and used for prioritization (Supplementary Fig. 1d, Supplementary Data 9 and Supplementary Note 1). Variants at 
a

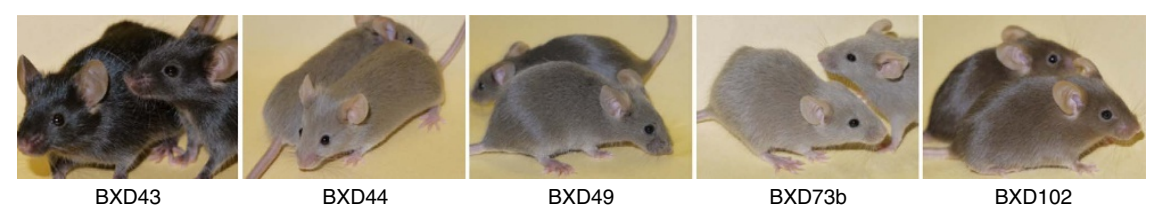

b

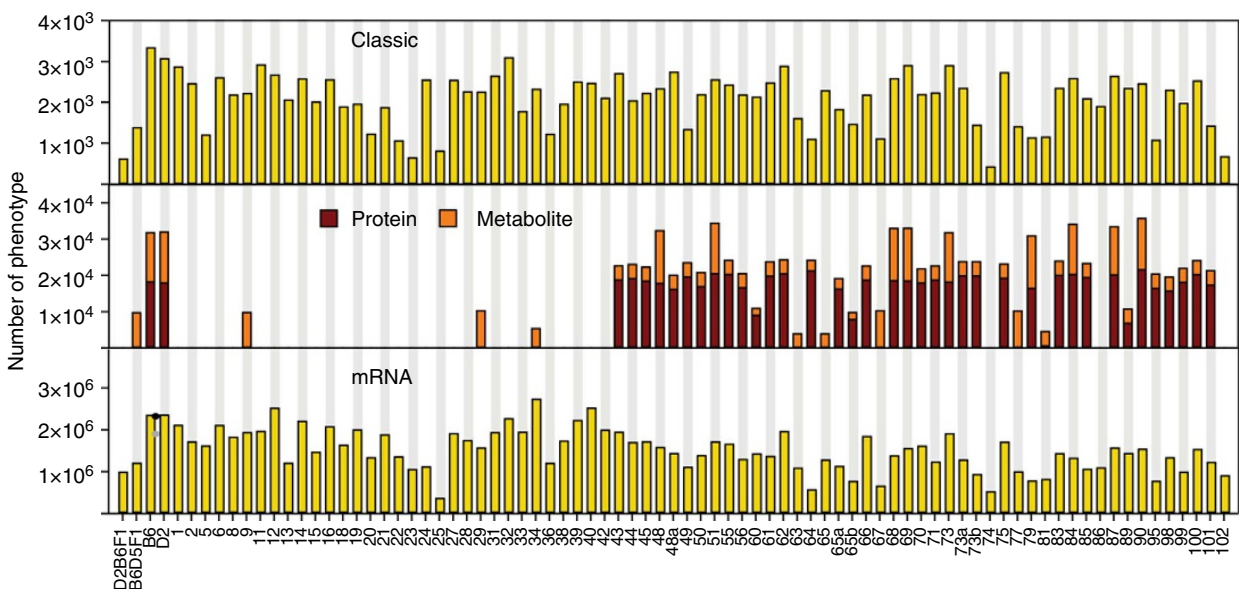

C
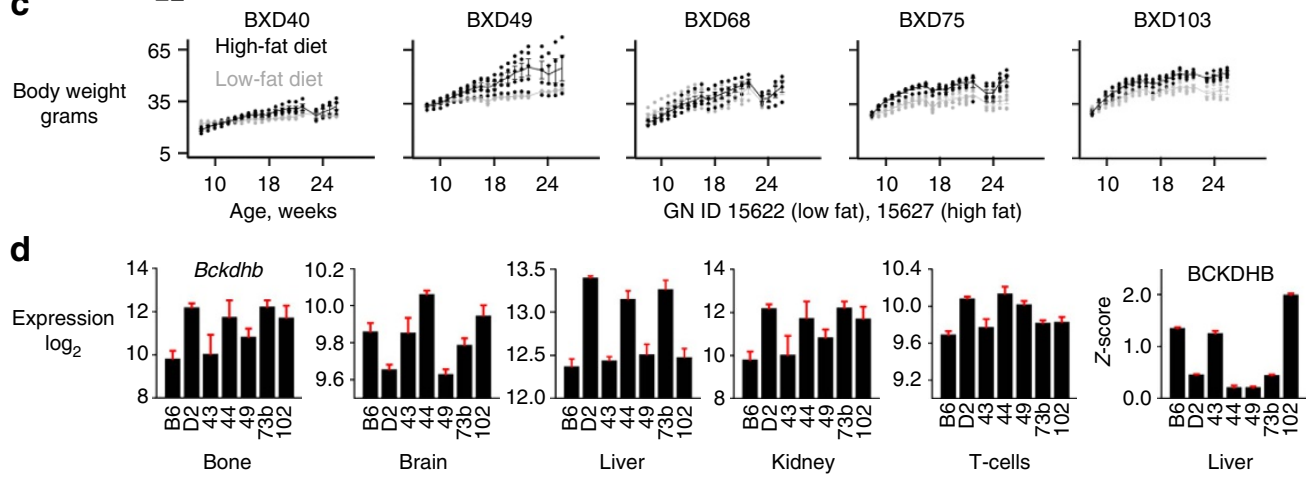

Figure 1 | Overview of phenome data for the BXD cohort. (a) Five pairs of isogenic BXD cohort strains-BXD43 to BXD102. There are now approximately 100 readily available $B X D$ strains and another 50 that are almost fully inbred. Almost all current phenome data is restricted to the parents, $\mathrm{F} 1$ hybrids (B6D2F1 and D2B6F1) and BXD1 through BXD102. (b) Phenome data categorized by type, including classic phenotypes (top and see comprehensive definitions in Supplementary Data 1), metabolic and proteomic trait data (middle), and independent mRNA expression assays (bottom, $n=86$ unique eQTL data sets, see Supplementary Note 1 Summary Expression Phenome). (c) Body weight data for BXD strains on high-fat (grey) and low-fat (black) diets taken from Wu et al. ${ }^{10}$. (d) Expression of Bckdhb mRNA and its protein in six tissues for the five BXD strains. Protein data from Wu et al. ${ }^{10}$

splice sites result in production of non-functional or abnormal proteins and are known to contribute to diseases ${ }^{13}$. We detected 70 SNPs that changed conserved bases at splice sites, including 29 acceptor sites (GT) and 41 donor sites (AG) (Supplementary Data 5). In addition, 26 of the ncSNPs are predicted to alter processed miRNA sequence (Supplementary Data 10 and Supplementary Note 1).

Insertions and deletions (indels) in coding sequences can be highly disruptive, especially when they introduce frameshift mutations. We found that most small indels (98.74\%) are in introns or intergenic regions, but 542 small deletions and 641 small insertions are in coding exons (Supplementary Fig. 2 and Supplementary Note 1). The small coding indels are enriched in trinucleotides, which account for $32 \%$ of small coding deletions and $38 \%$ of small coding insertions. Of the remaining coding indels, 45 are predicted to result in frameshift mutations through deletions (25) or insertions (20) (Supplementary Data 5).

Sequences from SOLiD and Illumina platforms were combined to accurately detect copy number variants (CNVs). We detected 16,817 CNVs, consisting of 4,296 gains and 12,521 losses (Fig. 2c) with an average length of 34.9 and $56.7 \mathrm{~kb}$, respectively (Supplementary Fig. 3). Of copy number gains relative to the
B6 genome, 79 cover 101 genes completely, while 300 cover one or more coding exons in 279 genes. Of the losses, 197 cover 259 genes completely, while 993 cover one or more coding exons in 276 genes.

All the sequence variants detected are summarized in Supplementary Data 11. FPR of each type of variants is shown in Fig. 2e, and detailed information is provided in Supplementary Note 1. The functionally important variants (that is, nonsense, missense, splice site, frameshift and CNVs) were selected for subsequent PheWAS analysis.

Phenome-wide association analysis in mouse. We used 3,805 genotypes that represent distinct haplotype blocks in the BXD family to perform PheWAS against 4,230 classic traits as well as 602,746 endophenotypic traits from 16 distinct tissues (Fig. 1a and Supplementary Data 1 and 2). This analysis yielded $\sim 14$ million genotype-to-phenome correlations and $\sim 2.0$ billion genotype-to-endophenotype correlations. A total of 95 genotypes are significantly associated with 321 phenotypes, corresponding to 108 phenotypic groups, at a stringent $q$ value threshold of $<0.01$ (Supplementary Data 12). In addition, we performed 

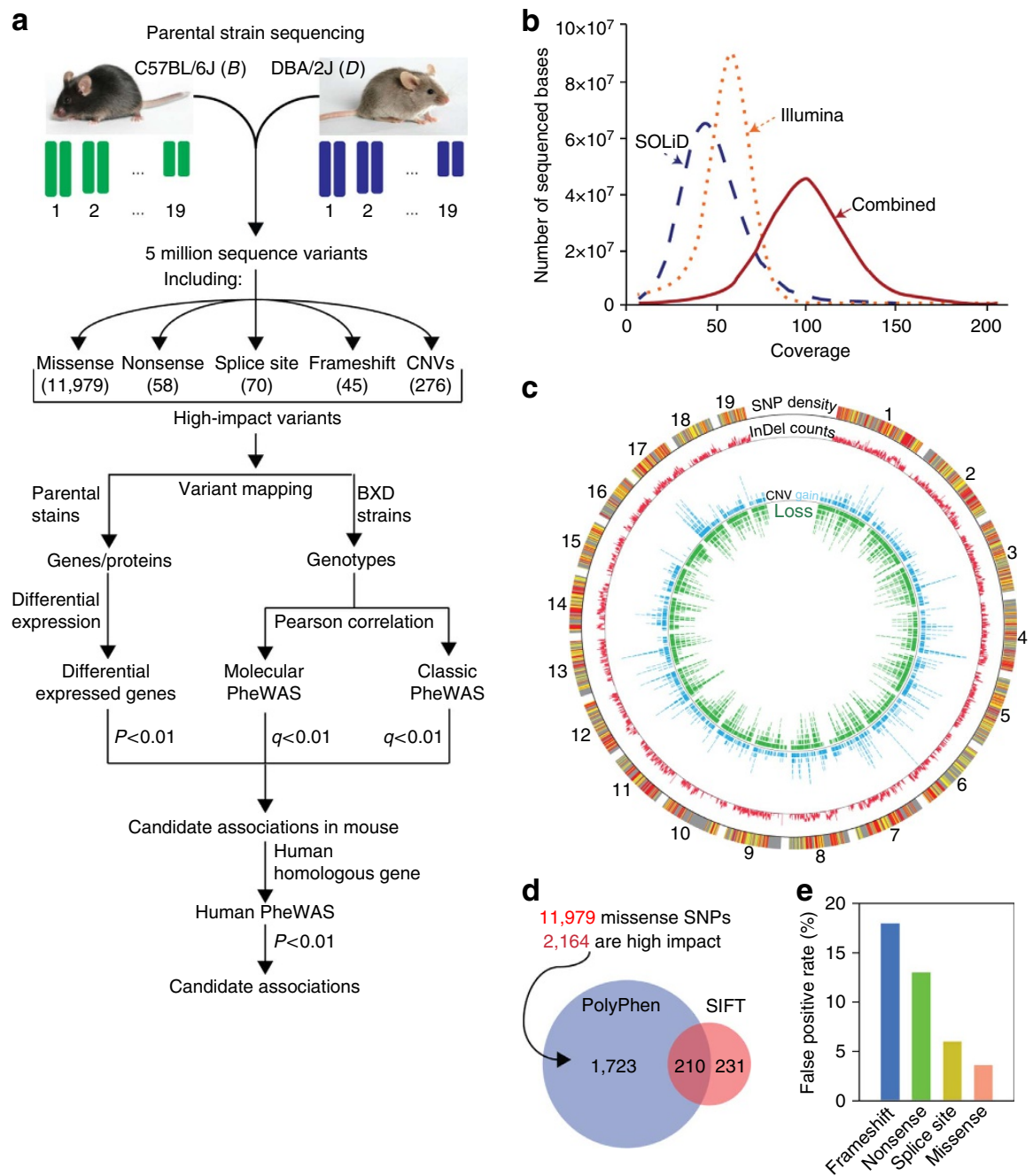

Figure 2 | Overview of experimental phenome-wide association. (a) Workflow for the PheWAS analysis. D2 genome was sequenced using Illumina and SOLiD platforms and aligned to the reference genome. Approximately five million sequence and structural variants were identified. A set of high-impact variants including 11,979 missense, 58 nonsense, 70 splice site, 45 frame shift and 276 CNVs spanning at least one gene was used for classic and molecular PheWAS analyses. (b) Whole-genome read coverage. The distribution was generated using combined mapped reads from Illumina and SOLiD, and mapped reads from Illumina and SOLiD platforms individually. (c) Circos plot of sequence and structure variants segregating in the BXD cohort. The outmost circle represents SNP density per $100 \mathrm{~kb}$ window (black at the lowest density and orange at the highest density). The second circle represents indel density per $100 \mathrm{~kb}$. The innermost circle represents CNVs. Blue and green ticks indicate D2 losses and gains, respectively. (d) Venn diagram of missense variants predicted to be deleterious by SIFT and PolyPhen2. (e) False positive rate of strong variants. A small subset of each type of variants was selected for validation by Sanger resequencing.

differential expression analyses between the B6 and D2 strains for each association by using transcripts from 16 tissues (Supplementary Data 2) and proteins from hippocampus (Supplementary Data 13).

We interrogated the associations for 12,420 functionally important variants, including 11,979 missense, 58 nonsense, 70 splice site and 45 frameshift mutations, and $276 \mathrm{CNVs}$, by mapping these variants to the nearest genotype markers within $\pm 1 \mathrm{Mb}$. We found that 650 functionally important variants were associated with 97 classic phenotypes, including 634 missense variants that were associated with 62 phenotypes (Supplementary Data 14).

Examples of variant-phenome association. Among 321 classic phenotypic associations meeting a stringent $q$ value threshold of $<0.01$ (Supplementary Data 12), a few variants, such as those in Gpnmb, Comt and H2-B1, have been associated previously with disease $\mathrm{e}^{14-17}$ using traditional forward genetics approaches
(Supplementary Data 15), but the vast majority of variants have not been previously linked to any phenotype. Here, we provided four PheWAS examples, including two missense variants (Fh1 and Col6a5), a nonsense variant (Ahr) and a CNV (a region covering both Alad and Hdh3). In addition, we also provided three other examples in the Supplementary Note 1, including a missense variant in Entpd2 (Supplementary Fig. 4), a noncoding variant in Hcfclr1 (Supplementary Fig. 5), and frameshift variant in Pcm1 (Supplementary Fig. 6).

The first example is a missense variant (A296T; $r$ s2536342) in the fumarate hydratase mitochondrial enzyme located on chromosome 1 at $175.60 \mathrm{Mb}$ (Fh1; Fig. 3a). Fh1 catalyses the hydration of fumarate to malate in the tricarboxylic acid (TCA) cycle and has been linked to renal cell cancer ${ }^{18}$. The missense variant in the lyase 1 domain is associated with a $\sim 1$.4-fold effect on expression of Fhl across many tissues, including midbrain, hypothalamus, striatum and spleen (Fig. 3b). This variant is strongly associated with Fh1 mRNA expression, as well as the 
a

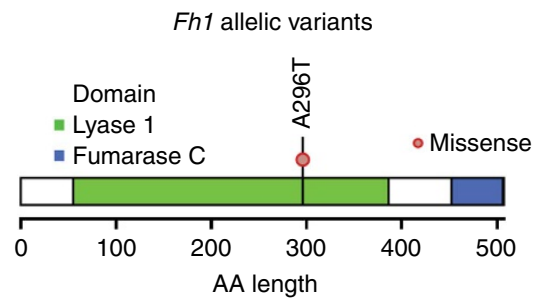

C

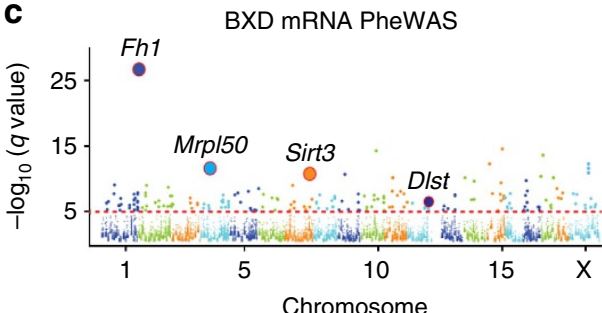

b

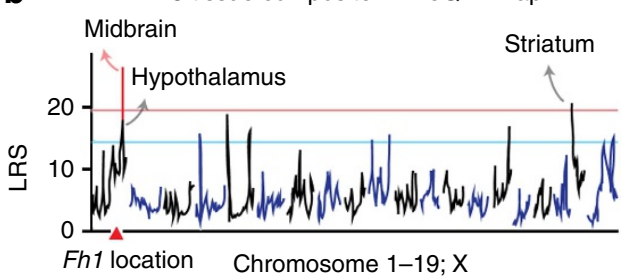

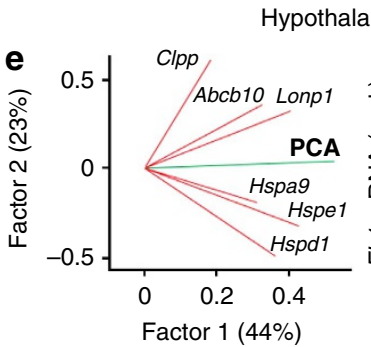
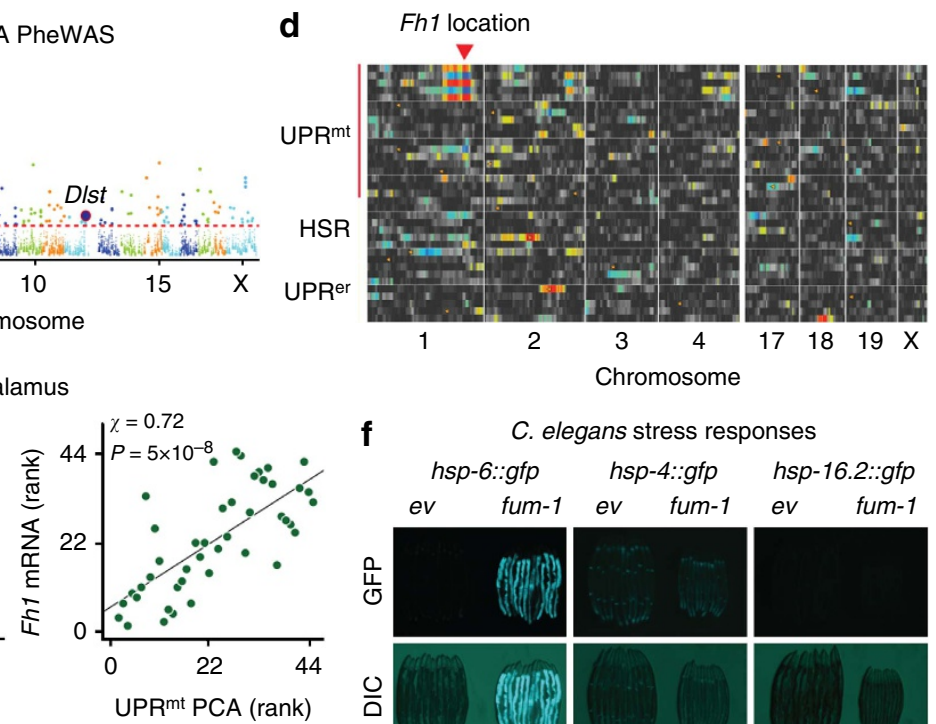

Fh1 location

f C. elegans stress responses
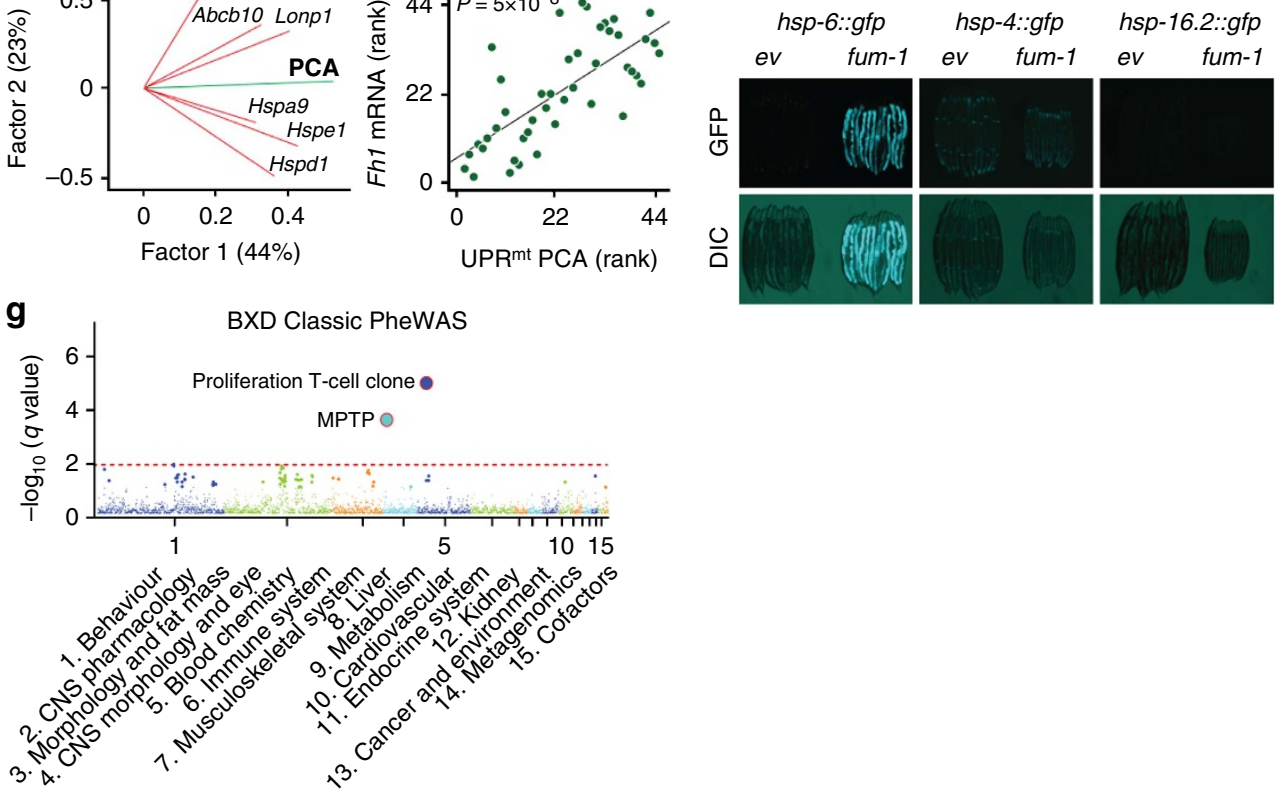

Figure 3 | Association analysis for a missense variant in Fh1. (a) Structure of the Fh1 gene showing lyase 1 and fumarase c domains, the former of which contains a missense mutation. (b) Combined eQTL mapping of Fh1 mRNA across 15 tissues. The eigenvalues associated with the first principal component map to Fh1 with a likelihood ratio statistic of $>20$. The solid red line represents genome-wide significance. The red triangle indicates the genomic position of Fh1. (c) Manhattan plot of an expression phenome scan of molecular traits linked to the Fh1 locus in midbrain. The $y$-axis shows the $-\log _{10} q$ values of association, and the $x$-axis shows positions of 55,681 assays generated using Agilent SurePrint array. (d) QTL heat map of mRNAs involved in the unfolded protein response. The $x$-axis lists mouse chromosome numbers. Each horizontal line represents the QTL map for a single transcript in midbrain. Transcripts are grouped into three major categories-genes involved in the canonical UPR ${ }^{\mathrm{mt}}$, cytoplasmic heat shock response (HSR) and unfolded protein response in the endoplasmic reticulum (UPR ${ }^{\mathrm{er}}$ ). A subset of UPR ${ }^{\mathrm{mt}}$ genes at the top are strongly modulated by the Fh1 locus on Chr 1 (the intense colours to the upper

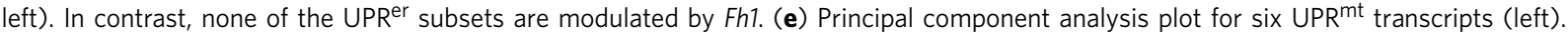

The first two components explain $\sim 67 \%$ of the variance in expression in hypothalamus. There is significant correlation between UPR ${ }^{\mathrm{mt}}$ expression and Fh1 $\left(P=5 \times 10^{-8}\right.$; Pearson product-moment correlation coefficient) (right). (f) Validation that fumarate hydratase selectively controls the UPR ${ }^{\mathrm{mt}}$ in $C$. elegans. The left-most pair of images demonstrates effects of the fum-1 RNAi knockdown on hsp-6::gfp signal-a marker of UPR ${ }^{\text {mt }}$ induction. The middle and right panels demonstrate that the fum-1 knockdown does not induce either the UPR ${ }^{\mathrm{er}}$ (hsp-4::gfp), or the cytoplasmic heat shock response (hsp-16.2::gfp). (g) Manhattan plot of a phenome scan. The $y$-axis shows the $-\log _{10} q$ values of $\sim 4,230$ phenotypes, and the $x$-axis shows the 15 phenotypic categories.

expression of other mitochondrial genes, including Mrpl50, Sirt3 and Dlst (Fig. 3c). Expression PheWAS shows that the Fh1 locus modulates mRNA expression levels of 113 mitochondrial proteins, in addition to eight genes linked to renal necrosis, and seven genes involved in mTOR signalling, consistent with the known role of FH1 in renal cancer (Supplementary Data 16).
Interestingly, four mitochondrial genes, Hspd1, Hspa9, Clpx and Lonp1 that all encode components of the mitochondrial unfolded protein response (UPR ${ }^{\mathrm{mt}}$ ) (ref. 19) - a still poorly characterized mitochondrial stress response pathway in mammals-show strong association with Fh1 (Fig. 3d and Supplementary Data 16). There is, furthermore, a significant correlation between Fh1 
transcript levels and principal component scores of a group of $\mathrm{UPR}^{\mathrm{mt}}$ genes in mouse (Fig. 3e). In contrast, no genes involved in the cytoplasmic heat shock response (HSR) or the ER unfolded protein response $\left(\mathrm{UPR}^{\mathrm{er}}\right)$ are associated with $F$ h 1 , indicating a selective association between $F h 1$ and $\mathrm{UPR}^{\mathrm{mt}}$ in mammals (Fig. 3d). To validate this association, we examined the phenotypic impact of the highly conserved Caenorhabditis elegans Fh1 ortholog, fum-1 (86\% sequence similarity) on unfolded protein responses. RNAi against fum-1 causes robust activation of the mitochondrial chaperone hsp- 6 induces green fluorescent protein (hsp-6::gfp) reporter, indicative of the activation of the UPR ${ }^{\mathrm{mt}}$ (Fig. 3f). The response was organelle-specific, and fum-1 RNAi does not induce either $h s p-4:: g f p$ or $h s p-16.2:: g f p$, reporters related to the $\mathrm{UPR}^{\mathrm{er}}$ or HSR, respectively (Fig. 3f). Thus, in the BXD family, a decrease of fumarate hydratase leads to a specific mitochondrial phenotype, characterized by an $\mathrm{UPR}^{\mathrm{mt}}$.

Fh1 is also associated with two candidate phenotypes: (1) T-cell proliferation (GN ID 10237; $q=2.6 \times 10^{-5}$ ), linked previously to mitochondrial function ${ }^{20}$; and (2) dopamine metabolism after treatment with the mitochondrial toxin 1-methyl-4-phenyl1,2,3,6-tetrahydropyridine (MPTP, GN ID 15151; $q=0.005$ ). Both traits are linked to $F h 1$ along with the control of mitochondrial components of a UPR ${ }^{\mathrm{mt}}$ pathway (Fig. $3 \mathrm{~g}$ ). No extant human genotype data are yet available for $\mathrm{FH}$-the homolog of Fh1.

The second example consists of a set of tightly linked missense variants in collagen $6 \mathrm{~A} 5$ on chromosome 9 at $105.76 \mathrm{Mb}$ (Col6a5, Fig. 4a). Col6a5 is a variant-rich gene and contains 21 missense variants, including a radical substitution (R1778C). Quantitative RTPCR shows higher expression of the $D$ allele than the $B$ allele in bone marrow (Fig. 4b). As expected, expression differences are strongly associated with the Col6a5 locus in bone expression PheWAS $\left(q=3.5 \times 10^{-4}\right.$, Fig. $\left.4 c\right)$. Unlike Fh1, the high density of linked variants in Col6a5 means that we cannot resolve effects of single SNPs, but the scan does define effects of $B$ and $D$ haplotypes for Col6a5 across the phenome. We find that this polymorphic gene is associated with differences in bone mineral density (BMD; GN ID 16532; $q=0.037$ ) (Fig. 4d) and quantitative micro-CT analysis confirms a marked difference in cortical BMD at the femoral midshaft between B6 $\left(1069.6 \pm 51.4 \mathrm{mgHA}\right.$ per $\left.\mathrm{cm}^{3}\right)$ and D2 parents $\left(1170.8 \pm 39.8 \mathrm{mgHA}\right.$ per $\mathrm{cm}^{3}$ ) (Fig. 4e,f). In humans, mutations in collagen VI are associated with a variety of musculoskeletal abnormalities ${ }^{21}$. We performed a matched PheWAS in human using the BioVU resource and linked $r s 113396273$ in exon 3 of COL6A5 (M56T) with osteopenia and other bone and cartilage disorders $\left(P=1.4 \times 10^{-3}\right.$; logistic regression; Fig. 4g). Like rs113396273, the other SNPs tested in COL6A5 demonstrated similar patterns of associations including respiratory abnormalities and giant cell arteritis.

The third example is a high-impact nonsense variant-a loss of stop codon in the $D$ allele of $A h r$. Ahr is an important transcription factor that modulates $\mathrm{P} 450$ gene expression in response to xenobiotics such as dioxin ${ }^{14}$. Although the effects of this SNP on protein length are already known ${ }^{22}$ (Fig. 5a), the pleiotropic consequences of this mutation have not been evaluated. This variant is significantly associated with mRNA $\left(q=1.7 \times 10^{-3}\right.$; Fig. $\left.5 b\right)$ and protein abundance of $A h r$ in liver $(q=0.0085$; Fig. $5 c)$. Classic PheWAS linked this variant to the frequency with which cleft palates is induced by 2,3,7,8-tetrachlorodibenzofuran injection (GN ID 10714; $q=3.2 \times 10^{-3}$ ) (Fig. 5c). Ahr variants have also been definitively linked to differences in locomotor activity ${ }^{17}$. Consistent with the results of the BXD PheWAS, a matched PheWAS in humans using BioVU links rs2066853 in AHR with cleft palate $(P=0.012$; logistic regression; Fig. $5 \mathrm{~d})$.
In the final example, we tested the effect of CNVs on gene expression and phenotypes. A CNV region on chromosome 4: 62.49-62.52 Mb that spans both Alad and Hdhd3-is interesting and involves a $4 \times$ expansion in strains with the $D$ haplotype. The $30 \mathrm{~kb} \mathrm{CNV}$ is otherwise identical by descent (Fig. 6a). This CNV is linked with high variation in mRNA expression of Alad and Hdhd3 in multiple brain regions (Fig. 6b,c), lung $\left(q=2.1 \times 10^{-7}\right)$, eye $\left(q=1.3 \times 10^{-10}\right)$ and liver $\left(q=9.2 \times 10^{-4}\right)$. Quantitative proteomics of hippocampus confirms significant upregulation (ALAD 2.3-fold, $P<0.01$, HDHD3 1.5-fold, $P<0.01$, see Supplementary Data 13). The CNV expansion of Alad and Hdhd3 is strongly linked to two classic phenotypes: pain response (GN ID 11307; $q=7.8 \times 10^{-3}$ ) and deoxycorticosterone levels in cerebral cortex (GN ID 12568; $q=2.6 \times 10^{-4}$ ) (Fig. 6d). A matched phenome scan in human demonstrates that $r s 1800435$ in $A L A D$ is associated with chronic pain $\left(P=2.2 \times 10^{-2}\right.$; logistic regression) (Fig. 6e).

Phenotypic resilience. One surprising finding is that a large proportion of genes with variants that we initially believed would have high phenotypic impact failed to associate with any classic phenotypes, or even with molecular endophenotypes. Among 41 confirmed nonsense variants with high predicted impacts, 18 nonsense variants failed to associate with any endophenotypes (across scans of 16 transcriptome data sets in different tissues) or with classic phenotypes at $q<0.01$. However, complete inactivation of four of these genes-Scn5a, Aimp1, Peli3 and Dlgap5-is known to cause severe phenotypes (MGI database, www. informatics.jax.org). Inactivation of $\operatorname{Scn} 5 a$ reduces embryo size and is associated with abnormal cardiovascular system function $^{23}$. Complete inactivation of Aimp1 produces delayed wound healing and decreased inflammatory response ${ }^{24}$. The other two genes with nonsense mutations, Peli3 and Dlgap5, are linked to decreased viral infection ${ }^{25}$ and female infertility ${ }^{26}$, respectively. Failure to detect associated phenotypes could be interpreted as false negative results or inadequate phenome coverage, but we suspect that most commonly this reflects molecular resilience that buffers the phenotype from apparently strong homozygous mutations. For example, a tandem duplication in the cardiac actin gene (Actc1) reduces expression of mRNA by $\sim 50 \%$ in hearts of strains that inherit the $D$ allele (Fig. 7a-c). This duplication upregulates expression of both skeletal muscle actin (Acta1) and smooth muscle actin (Acta2) by 30 and 50\%, respectively (Fig. 7c). Since variation in both of these actin transcripts maps precisely to Actc1, it is clear that this compensation is ultimately caused by the duplication (Fig. 7d,e). The depletion of cardiac actin has already been shown to be associated with compensatory increase in skeletal and smooth muscle actins in the mouse heart ${ }^{27}$. Another study has shown that Actc1 can effectively replace Acta1 to produce adequate function in the mouse postnatal skeletal muscle ${ }^{28}$.

\section{Discussion}

Recent work has demonstrated that phenome scans are a powerful way to link sequence variants to sets of phenotypes in clinical cohorts ${ }^{6,7}$. Here we have extended this approach to a murine cohort for which we have been generating cellular and molecular traits from many tissues and cell types and for which we can generate data on gene-by-environment interactions ${ }^{8,10,29}$. The variety and depth of phenotype data that we have assembled over the past decade for the BXD cohort make this the largest coherent multiscalar data set for any segregating population. Of course, there are an almost unlimited numbers of ways to extend this BXD phenome-from much more extensive geneenvironment interaction (GXE) studies to single-cell omics, but 
a

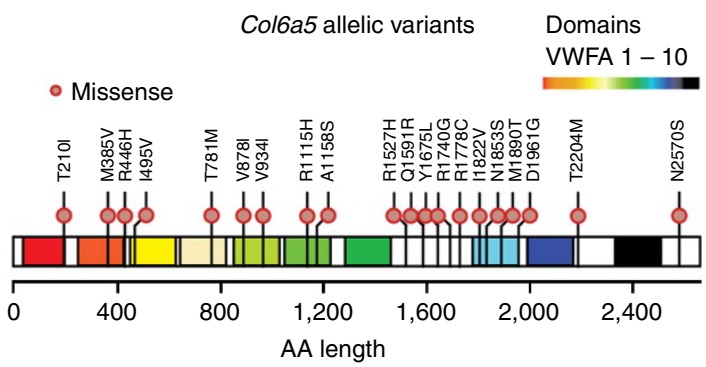

C

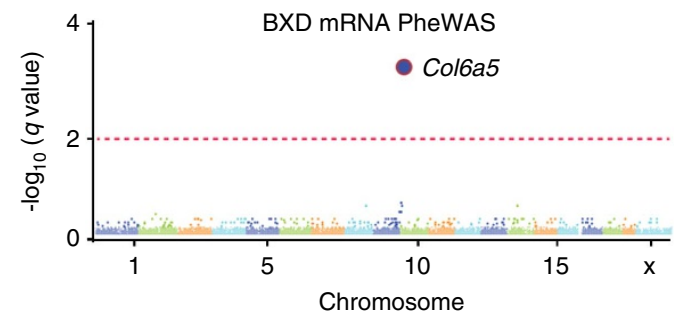

e

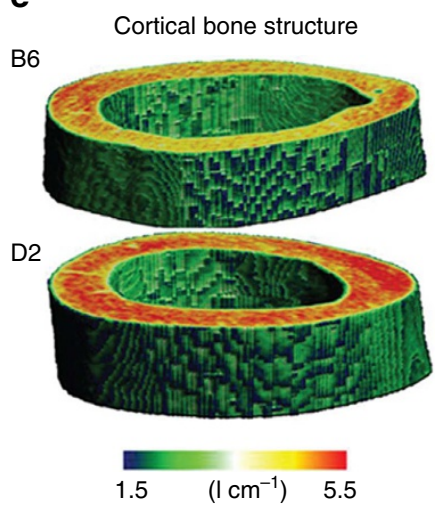

f

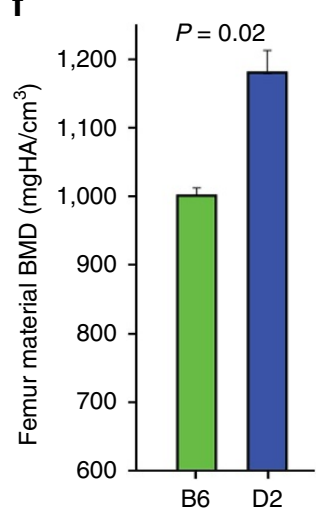

b

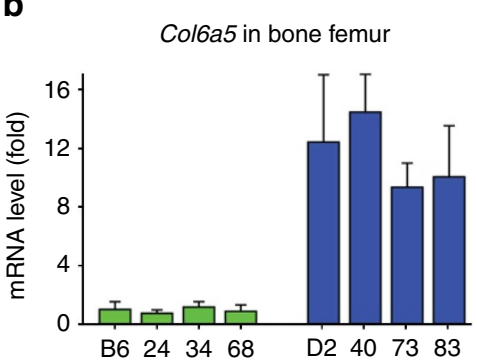

d

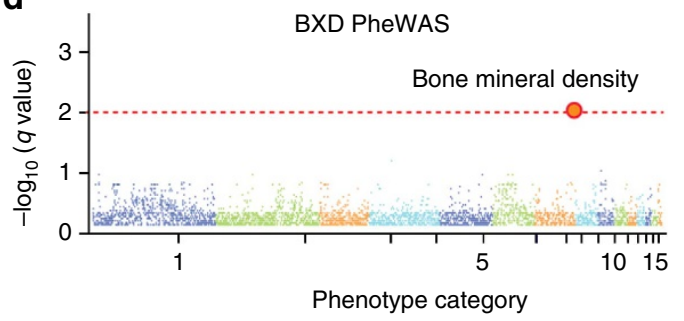

g

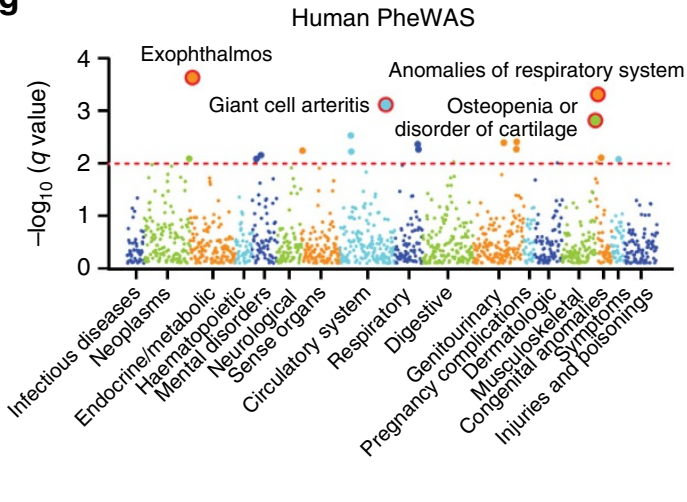

Figure 4 | Association analysis for missense variants in Col6a5. (a) Twenty missense variants in Col6a5 distributed across 10 von Willebrand factor A-type (vWFA) domains. (b) Differential mRNA expression of Col6a5 in tibias $(n=4)$ measured by rtPCR. The $D$ haplotype (blue, right) has far higher expression than the B haplotype (green) relative to Gapdh. (c) Phenome scan of Col6a5 (rs13480398) across mRNA assays for femur. (d) Phenome scan of Col6a5 (rs13480398) across classic phenotypes. (e) Marked difference in bone density between B6 and D2 parents. Femurs from 12-week-old mice were scanned using high-resolution micro-CT ( $\mu$ CT40, SCANCO Medical, Bassersdorf, Switzerland). More highly mineralized areas are indicated in red. (f) Difference in material bone density ( $P=0.02$; two-tailed Student's $t$-test, $n=3$ ). (g) Human phenome scan for association of Col6a5 ( $r$ 113396273) across BioVU.

at the current size, the phenome is certainly large enough to explore the utility of PheWAS in an experimental population. We demonstrate that phenome scans can be effective at linking sequence variants to a range of phenotypes and can be used to identify novel and unexpected genome-to-phenome relations, or to validate hypothesized associations from independent studies. Coupling mouse and human PheWAS cohorts also shows great promise, and provides an efficient method to validate and translate key genome-to-phenome relations.

The novel associations demonstrated in this study provide insight into the genetic basis of complex traits and variation in disease susceptibility. The missense variant in $F h 1$ is a case in point. A variant in $F h 1$ is linked to the UPR ${ }^{\mathrm{mt}}$, a protective stress pathway specific to mitochondria, and we confirmed that downregulation of fum-1, the C. elegans homolog of Fh1, activates the UPR ${ }^{\mathrm{mt}}$. Various disturbances have been shown to induce the UPR ${ }^{\mathrm{mt}}$, including treatment with paraquat, a pesticide that strongly induces reactive oxygen species ${ }^{30}$, activation of mitochondrial biogenesis ${ }^{31}$, overexpression of aggregation-prone mitochondrial proteins ${ }^{32}$ and interference with electron transport chain protein expression and assembly ${ }^{19,33}$. Here, we show that a purely metabolic perturbation, such as induced by loss of function of the TCA cycle component, fumarate hydratase, can activate the $\mathrm{UPR}^{\mathrm{mt}}$. While we have detected a single missense variant in Fh1, the molecular cascade that links Fh1 to other TCA cycle genes (that is, Dlst, $S d h a$ and $S d h b$ ) and a $\mathrm{UPR}^{\mathrm{mt}}$ proteostasis regulatory loop requires additional analysis.

Despite strong functional effects of variants in humans, the minor allele frequencies are often too low to attain sufficient sample size. Murine populations such as the BXDs, the Collaborative Cross and heterogeneous stock typically have linkage disequilibrium that is at least an order of magnitude larger than in humans. Consequently, the assignment of specific causality may be erroneous. For example, in the BXD family $\sim 20,000$ protein coding genes and 12,000 coding variants are distributed across $\sim 4,000$ haplotype blocks. Increasing the size and genetic diversity of a reference population and the number of recombination events can improve genetic resolution, but a more effective and meaningful solution, exemplified in this study, is to exploit other mouse cohorts and human cohorts for validation and cross-species translation. For example, by having multiple phenomes for a single species, along with matched databases of 
a

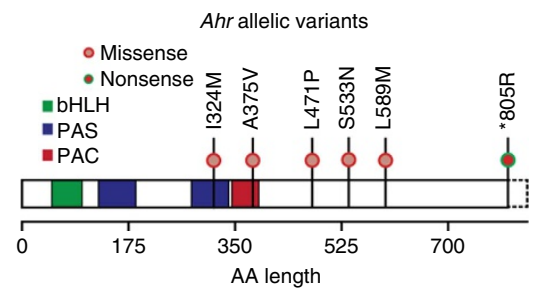

C

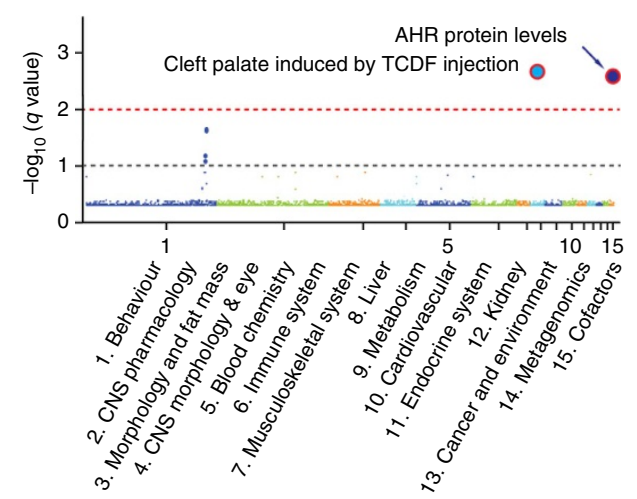

b

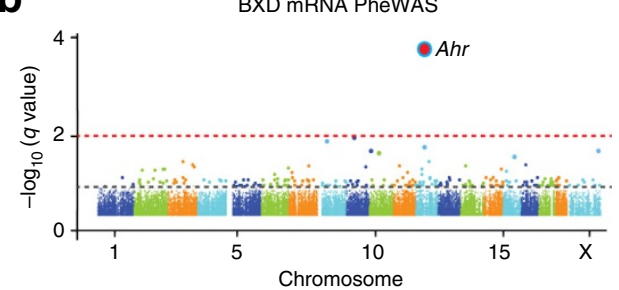

d

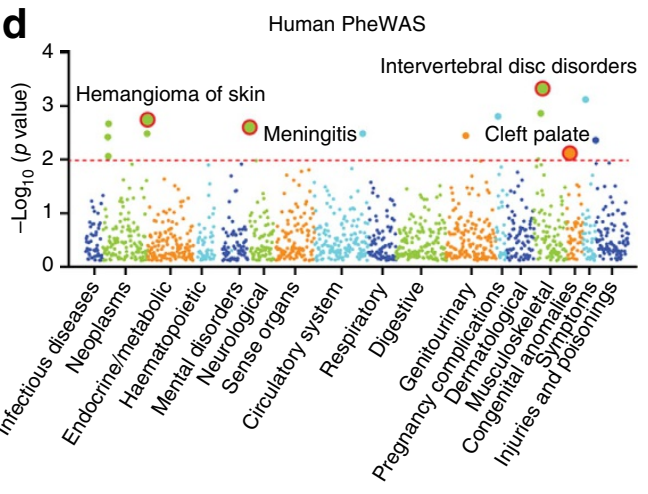

Figure 5 | Association analysis for nonsense variant in Ahr. (a) Structure of the Ahr gene showing three domains with a nonsense mutation and five missense mutations. The nonsense mutation ( ${ }^{\star} 805 R$ ) leads to loss of the stop codon, and the addition of $43 \mathrm{C}$-terminal amino acids. Dotted rectangle to the right is the extended coding region in the $D$ haplotype. (b) Expression phenome scan of Ahr ( $r$ 3711448) across liver mRNA levels in the BXDs. (c) Phenome scan of Ahr across classic phenotypes in the BXD strains. Both AHR protein level and cleft palate induced by TCDF injection are strongly linked to Ahr. (d) Manhattan plot showing the association in human between SNP (rs2066853) in AHR and classic phenotypes. The cleft palate phenotype is also associate with $A H R$ in human clinical cohorts.

segregating sequence variants, it would become practical to rapidly test the replicability of genome-phenome relations. It may soon be practical to compare the BXD phenome with that of the Collaborative Cross and other large families of RI strains. Any cohort will only segregate for a subset of possible sequence variants, and variants will often not be shared across populations or species. For this reason, conservation of gene function will be a more useful currency of exchange ${ }^{34,35}$.

While PheWAS has great potential, this approach faces several hurdles to more widespread application. The first is simply the technical and logistical challenge of generating a phenome. Intense collaborative efforts are a sine qua non even for the most tractable model organisms such as Drosophila ${ }^{36}$. The second is yet another example of the multiple testing problem: what is the appropriate correction given the size of the phenome and its structure? We have computed false discovery rate (FDR) $q$ values at a conservative threshold and have aligned our results, when possible, with the BioVU clinical cohort. However, in both species, the selection of appropriate $q$ values will depend on the purpose of studies and the relative costs of types 1 and 2 errors. Effective solutions may require adjusting thresholds based on the scope and intent of studies, as well as prior information about gene-to-phenotype relations. Alignment of phenotype associations across both humans and mice, however, adds validity to both. Very large, densely genotyped or sequenced populations will be needed to more deeply interrogate the human phenome. The third problem is linkage disequilibrium. The intervals in which sequence variants are located is a critical factor in mapping its phenotype spectrum. Pleiotropy will be inflated as a function of gene density, SNP density, and haplotype block structure. Deconvolving contributions of linked polymorphisms will, in most cases, still require independent experimental validation and, when possible, PheWAS of human cohorts.
We searched for molecular and functional consequences of $\sim 12,000$ coding variants, and were surprised that only a small fraction had strong effects on mRNA and protein expression, let alone on classic phenotypes. Is this resilience real or not? One obvious factor contributing to apparent phenotypic resilience may be inadequate depth of the phenome. Phenotypes may be detected only under specific conditions. For example, effects of the mutation in $\mathrm{Nnt}$ are much more pronounced under metabolic stress ${ }^{10}$. Artifactual resilience may also be caused by the presence of neighboring in-frame stop codons or splice acceptor or donor sites. For example, the loss of a stop codon in Dlgap5 only adds two amino acids due to the presence of a tandem stop codon six nucleotides downstream. Additionally, some negative results are likely to be caused by incorrect or incomplete gene models that generate spurious high impact variants.

In contrast, genuine phenotypic resilience is likely to result from functional overlap and compensation among paralogs and other members of complex molecular networks. Even after stringently filtering both sequence data and gene models, it is clear that many strong sequence variants are successfully buffered at intermediate levels ${ }^{37-39}$. For example, a splice site mutation in Cyp2c39 (Supplementary Table 5) inactivates this P450 enzyme but has no detectable impact on higher order phenotypes-a compelling negative result. An obvious explanation is overlap with other members of the Cyp2c cluster. The strongest exemplar of paralog buffering in our study is the mutation in Actc1, which causes compensatory upregulation of the expression of both Acta1 and Acta2 (Fig. 7). In retrospect, this buffering of genetic variation is not surprising. A large fraction of knockout mutations in mice and other well-typed species are viable and many of these do not have any known functional consequences ${ }^{40,41}$.

The combination of deep phenotyping and full genome sequencing makes it possible to reverse the polarity of 

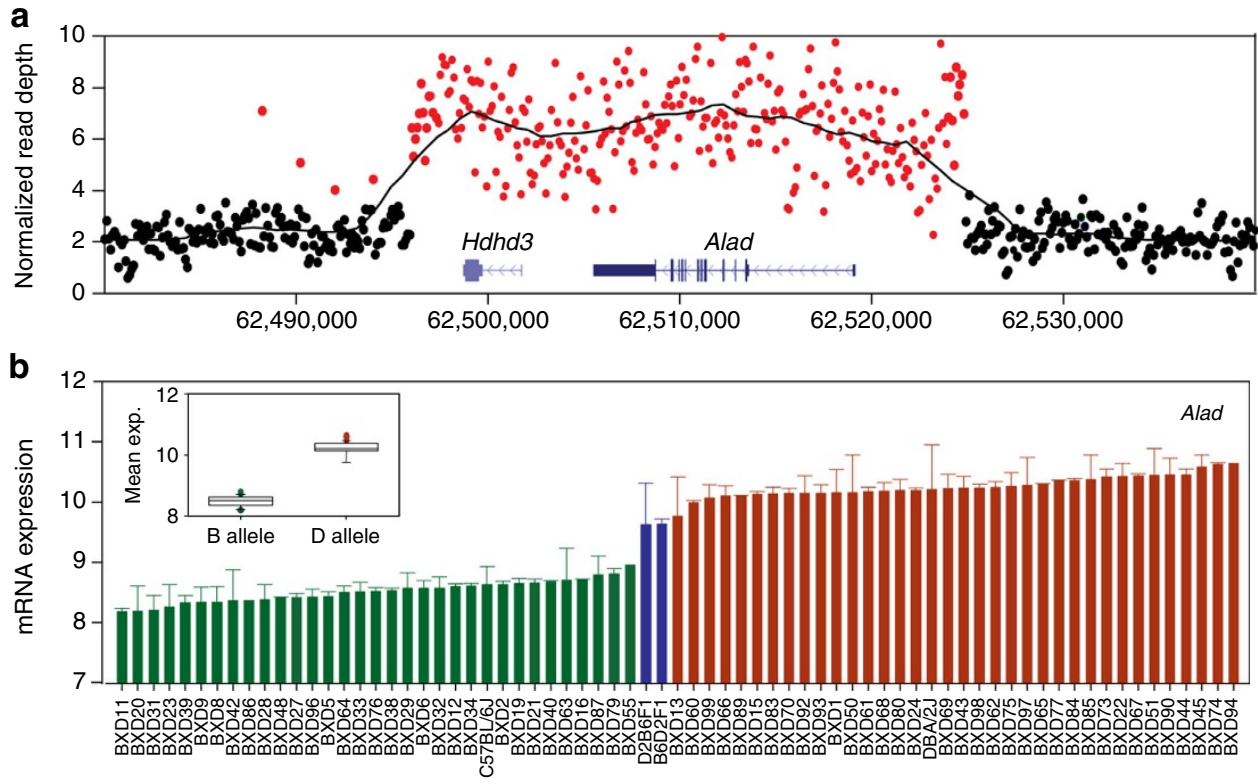

C
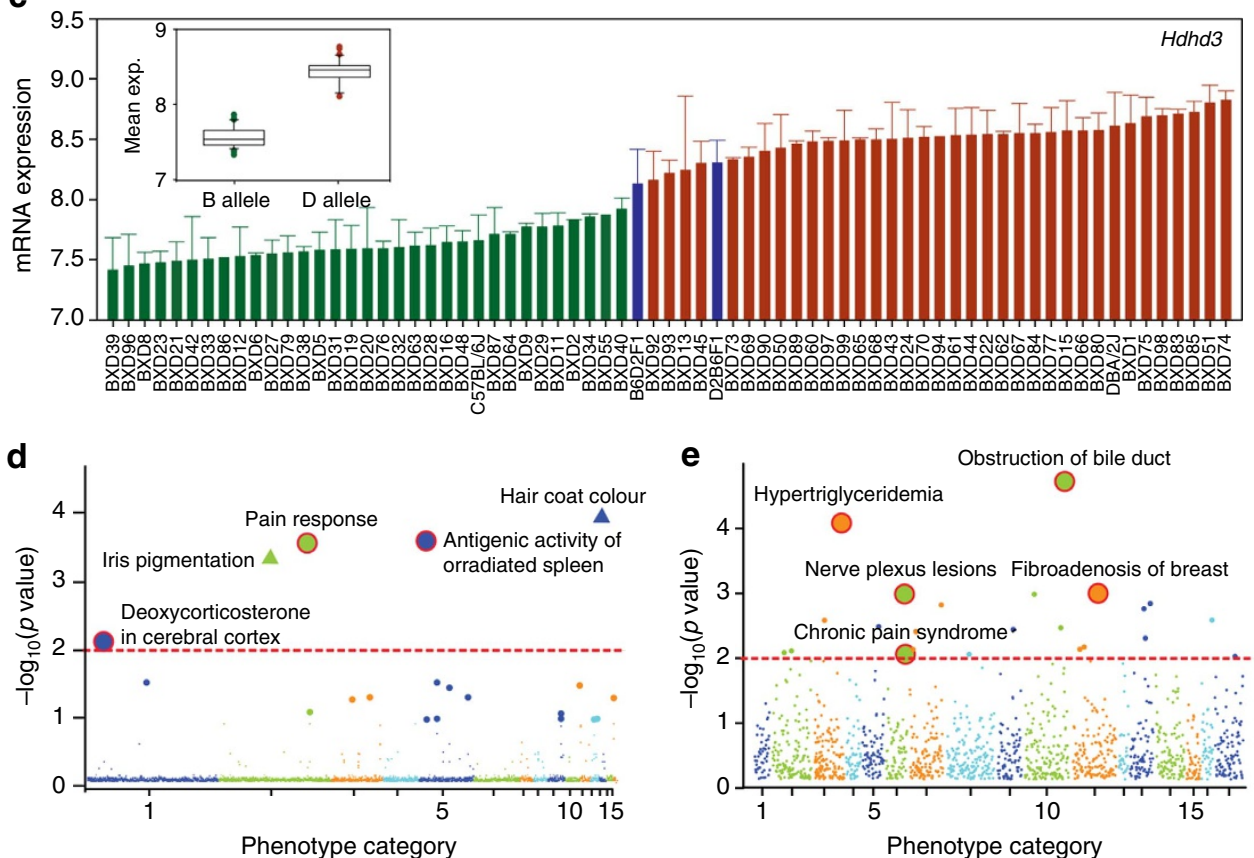

Figure 6 | Association analysis for CNV covering Alad and Hdhd3. (a) The CNV region for Alad and Hdhd3 derived using read-depth information from genome sequencing. Red dots represent at least a two-fold increase in coverage compared with the reference genome. The $x$-axis shows the reference genomic position of the CNV. Two gene models (that is, Hdhd3 and Alad) are shown in the CNV plot. (b,c) Rank ordered mean expression levels of Hdhd3 and Alad across 67 BXD strains, their parental strains, and F1 crosses. Expression values are normalized on a log2 scale (mean \pm s.e.m.). Strains with $D$ alleles (red) have higher levels of Alad and Hdhd3 compared with $B$ alleles (green). F1 hybrids (blue) are intermediate. The comparison between $B$ and $D$ alleles for Alad and Hdhd3 are shown in an inset boxplot. (d) The phenome scan of the BXD cohort highlights several interesting potential phenotypes including pain response (thermal nociception), brain deoxycorticosterone levels, and antigenic activity in the spleen. Two triangles represent pigmentation traits that we know are associated with a variant in the linkage disequilibrium block. (e) Manhattan plot obtained after phenome scan of the BioVU EHR data showing the association in humans between a SNP (rs1800435) in ALAD and chronic pain syndrome and several other classic phenotypes.

genome-wide analysis and to measure the impact of defined sequence variants at many levels to biological organization-from mRNAs to metagenomic profiles and behavioural variation. The combination of experimentally tractable murine resources, such as the BXD family, with human clinical cohorts such as BioVU, is an efficient and scalable way to validate and translate genes to the linked sets of phenotypes. Even negative results can be genuinely informative, essentially an inverse of missing heritability.

\section{Methods}

DNA sample for sequencing. DBA/2J foundation breeding stock at generation F223 was obtained from The Jackson Laboratory (www.jax.org). Genomic DNA was isolated from livers of filial generation F224 female littermates using a QIACube and DNeasy kits (Qiagen Inc., Valencia, CA).

All work with mice as conducted under a protocol approved by the UTHSC institutional animal care and use committee.

Libraries preparation. Six libraries were constructed. Three mate-paired libraries with insert sizes of 1,2 and $3 \mathrm{~kb}$ were prepared for ligation-based sequencing 

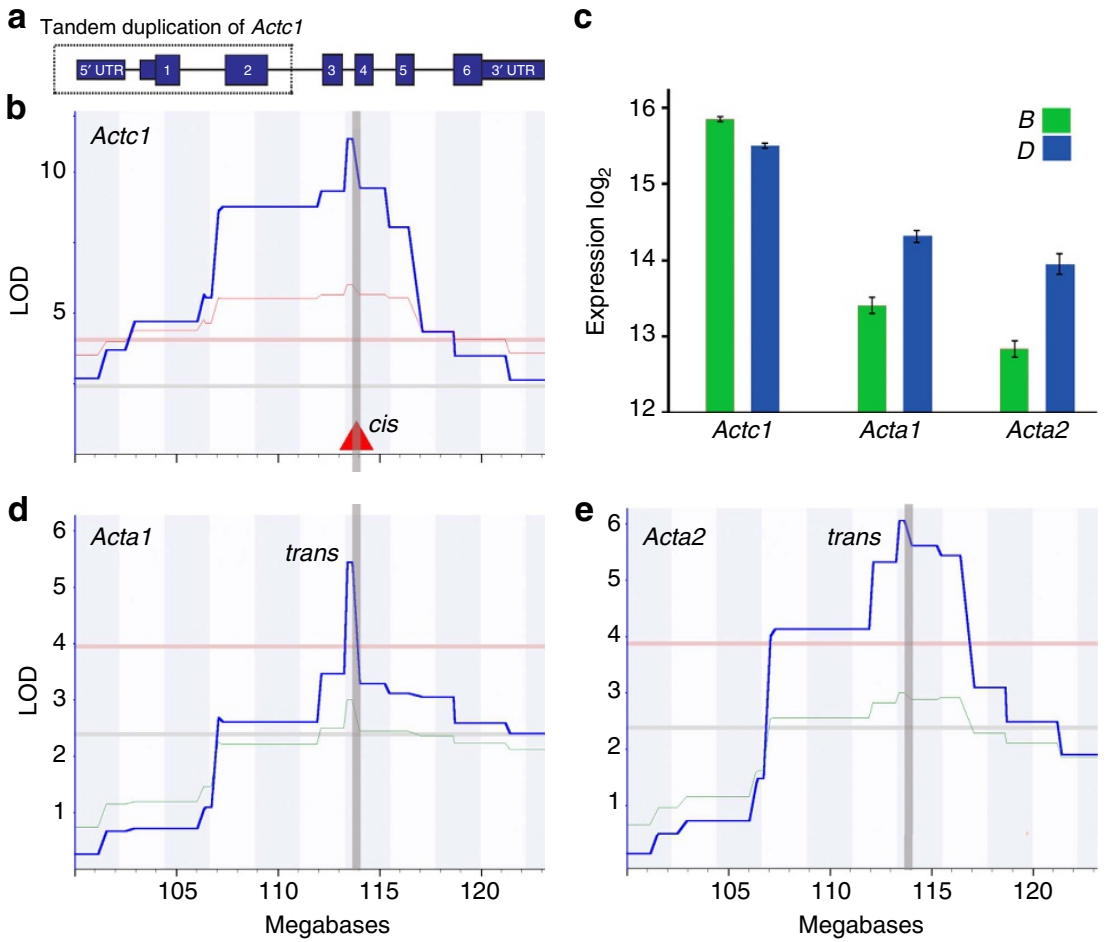

Figure 7 | Compensation for Actc1 loss by upregulation of Acta1 and Acta2. (a) Structure of the cardiac actin gene, Actc1, and the tandem duplication (dotted box) in strains with the $D$ haplotype. (b) Actc1 expression in heart is controlled by a strong cis-eQTL that corresponds precisely to the location of the Actc1 gene on Chr 2 at $114 \mathrm{Mb}$ (solid red triangle). $X$-axis represents the megabase coordinate on Chr 2 while the $y$-axis represents the LOD linkage score. Thin horizontal lines provide genome-wide significance thresholds (upper red line at $P<0.05$ and lower grey line at $P<0.63$ ). (c) Mean expression of Actc1, Acta1 and Acta2 highlights the partial compensation for $B$ haplotypes (green, $n=24$ ) and $D$ haplotypes (blue, $n=12$ ). Expression values are normalized on a log2 scale (mean \pm s.e.m.). Heart expression data is from GeneNetwork data set GN485 (EPFL/LISP BXD CD-HFD Heart Affy Mouse Gene 2.0 ST (Jan14) RMA). (d,e) Both skeletal muscle and smooth muscle actins (Acta1 and Acta2) also map precisely to Actc1.

(ABI SOLiD 2 and 3). Three paired-end libraries with insert sizes between 175 and $215 \mathrm{bp}$ were prepared for sequencing by synthesis (Illumina GAII and HiSeq 2000). Standard protocols recommended by the manufacturers were used in all cases.

Sequencing. Three mate-paired libraries were sequenced using ABI systems according to the vendor's protocol. Three libraries were sequenced using reagent kit V4 and Illumina systems according to the manufacture's standard protocol. Read lengths for the SOLiD2 and SOLiD3 systems were $2 \times 25$ bp and $2 \times 50$ bp, respectively.

One library was sequenced using the sequencing reagent V4 on the Illumina HiSeq 2000 following the manufacture's standard cluster generation and sequencing protocol. Sequence reads with $101 \mathrm{bp}$ on both ends were extracted from images files using the GA software (version 1.4).

Sequence alignment. SOLiD reads were aligned to the B6 reference genome using two alignment tools: Corona Lite v4.0.2 (http://www.thermofisher.com/us/en/ home/technical-resources/software-downloads/solid-software.html) and the MAQ code v0.7.1 (ref. 42). Corona Lite parameters were set to allow up to two and six mismatches for 25 and $50 \mathrm{bp}$ sequence fragments, respectively. The longer reads from Illumina were mapped to the reference genome using $\mathrm{MAQ}$, allowing up to two mismatches in the first $24 \mathrm{bp}$ of each read. All alignment files were converted into BAM format. Reads that aligned to only one location with no more than two mismatches in the first $24 \mathrm{bp}$ were considered uniquely aligned.

SNP detection. ABI Bioscope software was used to align colour-space reads and to detect SNPs against the B6 reference genome. Similarly, MAQ software was used to align reads and to identify SNPs. A threshold of three or more supporting reads and a consensus quality score $>30$ (Illumina) and a confidence value $>0.5$ (SOLiD) was used to declare SNPs. Rare heterozygous calls, presumably generated by alignment errors, were discarded.

Indel detection by mapped and unmapped reads. Four tools were used to identify indels-Corona Lite small indels pipeline, MAQ, Pinde ${ }^{43}$ and BreakDancer ${ }^{44}$. Corona Lite was used to identify insertions of up to $3 \mathrm{bp}$ and deletions of up to $11 \mathrm{bp}$ using SOLiD sequence. Indels were further filtered by requiring at least three supporting reads, and indels within regions having extremely high coverage $(1000 \times)$ were excluded. MAQ was also used to identify insertions up to $19 \mathrm{bp}$ and deletions of up to $86 \mathrm{bp}$ for $101 \mathrm{bp}$ Illumina reads. A threshold of a minimum of three supporting reads and a minimum consensus quality of 30 was used. Rare heterozygous indels were discarded. Pindel was used to identify indels from 1 to $100 \mathrm{~kb}$. Access to all of these variants is available on a custom version of the UCSC Genome Brower at http:// ucscbrowser.genenetwork.org/cgi-bin/hgGateway.

Large deletions detected by mapping distance. $\mathrm{AB}$ large InDel Tool (v1.0), which identifies deviation in clone insert size, was used to detect large indels from SOLiD reads. Indels were supported by at least two clones (cluster of reads) and a distance deviation greater than 6 s.d. from the normal distribution of insert size. Breakdancer ${ }^{45}$ was used to detect large structural indels (100-100,000 bp) from Illumina reads. The distribution of insert size from high quality $(>35)$ paired-end reads was computed, and those greater than 6 s.d. from the mean were used to define large indels. A minimum confidence score of 90 was used by BreakDancer. All clones that deviated by more than $100 \mathrm{~kb}$ were discarded for both methods.

CNV detection. Before performing CNV detection, we converted mapping data into the BAM format and removed gaps in the reference genome. CNVs were detected using an event-wise testing method based on read depth ${ }^{46}$. This method estimates the coverage of read depth in $100 \mathrm{bp}$ non-overlapping windows and then performs significance testing. In this study, CNVs were defined as having at least 10 consecutive windows (100 bp each) with a minimum size of $1 \mathrm{~kb}$. Merged events were filtered stringently at a significance level of $10^{-6}$.

Genes affected by sequence and structural variants. We examined the impact of SNPs, indels, inversions, and CNVs located within annotated gene models (ENSEMBL version 60). Each variant was evaluated for overlap with gene models and annotated as $5^{\prime}$ UTR, coding/exon, intron, $3^{\prime}$ UTR and intergenic regions using in-house Python scripts (available upon request). Sequence variants were further examined and classified by types of variants, including nonsense, missense, splicing variant and frameshift. When possible, variants were also annotated by gene symbol and other accessions. 
Generating impact scores for coding and noncoding SNPs. We used two different methods-SIFT and PolyPhen-to predict whether amino acid substitutions in coding SNPs are likely to affect protein function. SIFT uses sequence homology and the physical properties of amino acids to predict impact, while PolyPhen uses sequence-based and structure-based predictive features.

The impact scores for ncSNPs consist of two parts: a functional element score and a mutation effect score. The functional element score reflects how likely it is that the SNP is located in a functional region of the genome, and the mutation effect score estimates how likely a mutation at this position is to affect the function of an overlapping region. Any ncSNP that overlaps a known functional element in the UCSC Mouse Genome Browser (mm10, GRCm38 assembly38) receives a functional element score of 1 . Functional elements are defined using the following gene regulation tracks: open regulatory annotation (ORegAnno) elements, the NHGRI's bi-directional promoters, and Repressor Element 1-Silencing Transcription Factor (REST) binding sites, as well as conserved elements as predicted by the phastCons program (conservation track) (ref. 47). Furthermore, ncSNPs overlapping a predicted conserved element were assigned the element's phastCons score. The mutation score is based on the evolutionary conservation of the position in alignments with other placental mammals. The phyloP package (ref. 48) was used to estimate conservation. The ncSNP impact score is the product of the functional element and mutation effect scores. If the data required to compute the two scores were missing for one, but not the other, then the missing score was assigned a non-zero value less than the scores observed for all positions with data present. This prevents evidence for the functional relevance of a SNP from one source from being completely ignored if the other is not present. Thus, all ncSNPs with non-zero impact scores have some evidence of functional relevance.

\section{Comparative genomic analysis. Orthologs of murine proteins from complete} genomes of 77 higher eukaryotes were obtained using BLAST searches. Genomes containing paralogs of a given gene were excluded from consideration. Multiple sequence alignments of orthologs were constructed using the LINS-I algorithm in MAFFT $^{49}$. Amino acid substitutions in a position corresponding to a given SNP were analysed. SNPs that occur in invariable positions in mammals were considered likely to be deleterious. Non-mammalian conservation was considered as enhancing prediction strength. SNPs that occur in variable positions in mammals were considered unlikely to be deleterious. Variable positions in rodents and primates were also considered unlikely to be deleterious.

Experimental validation for SNPs. SNPs and indels were selected for validation by traditional Sanger sequencing. Primers were designed using Primer3 (http:// frodo.wi.mit.ed/primer3/). PCR assays were performed using $5 \mathrm{ng}$ DBA/2J genomic DNA, $10 \mathrm{pmol}$ each of forward and reverse primer in $50 \mu \mathrm{l}$. The following cycle parameters were used: $95^{\circ} \mathrm{C}$ for $4 \mathrm{~min} ; 35 \mathrm{cycles}$ of $95^{\circ} \mathrm{C}$ for $30 \mathrm{~s} ; 55^{\circ} \mathrm{C}$ for $30 \mathrm{~s}$ and $72{ }^{\circ} \mathrm{C}$ for $1 \mathrm{~min}$; and $72{ }^{\circ} \mathrm{C}$ for $5 \mathrm{~min}$. PCR products were purified with $2 \mu \mathrm{l}$ ExoSAP-IT (Invitrogen Corporation). Sanger sequencing was performed using an $\mathrm{ABI} 3730$.

Experimental validation of indels. A total of 40 indels were selected from each chromosome for validation. These included 20 small- to medium-sized insertions ( $<86 \mathrm{bp}$ ), 20 medium-sized deletions (size $>50 \mathrm{bp}$ ), and 20 large deletions ( $>100 \mathrm{bp}$ ) detected by Illumina GA2. Primers were designed by centring the target indel to produce amplicons that were $300-400$ bp in length using Primer 3. PCR assays were performed as described above. We ran PCR-amplified genomic DNA on SDS-polyacrylamide gel electrophoresis gels. Each PCR assay was performed on DNA from B6 and D2. The sizes of the resulting PCR products were compared with the predicted size of indels.

Detection of SNPs in Affymetrix probe-binding regions. To find probe sequences affected by variants (SNPs and small indels) within probe binding regions of the Affymetrix Mouse Genome 4302.0 array, we mapped all probes to the mouse reference genome using Blat. Probe regions were then examined for the presence of SNPs or small indels.

Proteome-wide quantification between the B6 and D2 strains. Adult B6 and D2 strains were used for protein quantification with three biological replicates of hippocampus. The hippocampal tissues were dissected as previously described ${ }^{50}$ and lysed in lysis buffer ( $8 \mathrm{M}$ urea, $0.5 \%$ sodium deoxycholate, $50 \mathrm{mM}$ HEPES and $\mathrm{pH}$ 8.5). In short, fixed brains were bisected along the midline. Left and right hippocampal regions were dissected under a dissecting microscope by inserting fine blunt forceps into the ventricular cavity just dorsal to the hippocampus and removing overlying cortex and callosum. The surface of the hippocampus and dentate gyrus was used to guide removal of cortex along the septotemporal axis. The exposed hippocampus and dentate gyrus was pulled free of the hemisphere in a ventral-to-dorsal direction. The dorsoanterior aspect of each hippocampus was trimmed free of septum and dorsal fornix, rolled quickly in tissue paper, and immediately weighed to the nearest $0.1 \mathrm{mg}$. The dissection includes a small part of the subiculum adjacent to CA1 and occasionally a small strand of the fimbria. For each sample, $100 \mu \mathrm{g}$ of extracted proteins were digested with LysC $(1: 100, \mathrm{w} / \mathrm{w})$ for
$3 \mathrm{~h}$. Samples were diluted four times with $50 \mathrm{mM}$ HEPES followed by trypsin digestion $(1: 50, \mathrm{w} / \mathrm{w})$ overnight at room temperature. After digestion, the samples were acidified with trifluoric acid to a $\mathrm{pH}<2$ and desalted and dried in a speed vacuum. Peptides were labelled with six-plex TMT reagents (Thermo Scientific) as recommended by the manufacturer. Following labelling, the TMT-labelled samples (TMT126-TMT131) were mixed equally to generate a digest mixture which was further fractionated by high $\mathrm{pH}$ reverse phase liquid chromatography. Ten fractions are collected and further analysed by low $\mathrm{pH}$ reverse phase liquid chromatography-tandem mass spectrometry (MS/MS).

All digest mixtures were analysed on Q Exactive MS (Thermo Fisher Scientific) with one MS survey scan and up to 10 data-dependent MS/MS scans. The instrument was operated at a mass resolution of $35,000,1 \mathrm{e} 6$ automatic gain control target, and $60 \mathrm{~ms}$ maximal ion time for MS, and a mass resolution of 17500 , $10^{5}$ automatic gain control, $250 \mathrm{~ms}$ maximal ion time, $2 \mathrm{~m} / \mathrm{z}$ isolation window and $30 \mathrm{~s}$ dynamic exclusion time for MS/MS. The normalized collision energy was set to $28 \%$.

Raw data from each fraction were searched using SEQUEST (v28) against a mouse SwissProt/trEMBL database (release of 09/09/2011), concatenated with a decoy database with all the protein sequences in reverse order. Searches were performed using a 15 p.p.m. mass tolerance for precursor ions and $0.02 \mathrm{Da}$ window for fragment ions, allowing up to two missed trypsin cleavage sites. Six-plex TMT tags on lysine residues and peptide $\mathrm{N}$ termini $(+229.162932 \mathrm{Da})$ and oxidation of methionine residues $(+15.99492 \mathrm{Da})$ were used for dynamic modification, and carbamidomethylation of cysteine residues $(+57.021 \mathrm{Da})$ was used for static modifications.

Organization and categorization of mouse phenome. The BXD Phenotype database has been amassed by literature review, direct data entry by our team, and by collaboration with many investigators. Data are reviewed before entry in GeneNetwork by the senior author. Phenotypes are currently split into 15 broad phenotypic categories (Supplementary Data 1). Phenome curation and description was initiated by R.W.W. and Dr Elissa Chesler in 2002 by literature review and data extraction. The early work is described briefly in Chesler et al. ${ }^{51,52}$. Most work over the past 5 years has been performed by two of the coauthors (R.W.W. and M.K.M.). We have used a controlled vocabulary and set of rules described here (http://www.genenetwork.org/faq.html\#Q-22). Descriptions include a 'prefix' of major biological and domain categories such as 'central nervous system', 'cancer biology' and 'immune system'. These domains have been used to define major categories used in figures and graphs.

PheWAS analysis in mice. PheWAS were performed for a total of $\sim 12,000$ variants, including 11,979 missense, 58 nonsense, 68 splice site, 39 frameshift mutations and 276 CNVs The closest marker for each variant from a set of 3,804 genetic markers-each representing a unique haplotype block-was used to represent that variant in the PheWAS. We used 16 expression data sets representing different tissues of the BXD strains to explore the genetic basis of variation at mRNA levels. Similarly, we used 4,236 classic phenotypes from GeneNetwork.org (www.genenetwork.org) to study the association between variants and phenotypes. We calculated the $P$ value of the Pearson correlation between each marker (variant) and 4,236 phenotypes and $\sim 40,000$ transcripts for the expression data. All $P$ values of correlation were calculated as a two-tailed test, and the $q$ values (FDR) were calculated using QVALUE (ref. 53). We used an FDR threshold of 0.01 for associations. The analyses were performed using in-house Python scripts, and the R statistical package.

PheWAS in humans. PheWAS for human data was performed using 29,722 individuals with Illumina HumanExome array data identified as European ancestry in the EHR and by using structure ${ }^{54}$. To define diseases, we mapped International Classifications of Diseases, 9th edition, (ICD9) codes from the EMR into 1,645 possible PheWAS phenotypes using methods described previously ${ }^{6}$. PheWAS phenotypes aggregate like ICD9 codes together (for example, type 2 diabetes codes as a specific phenotype), are hierarchical (for example, 'inflammatory bowel disease' is a parent of 'Crohn's disease' and 'Ulcerative colitis'), and include logic to select controls for each case definition. We considered only phenotypes with at least 20 individuals for analysis, and required each case to have at least two ICD9 codes for a PheWAS phenotype to be considered a case (those with only one code are neither a case nor a control). Each SNP-phenotype association test was run with PLINK (ref. 55) using logistic regression adjusted for age, sex and the first three principal components as calculated by EIGENSTRAT using ancestry informative markers. Analysis was performed assuming an additive genetic model. These data were aggregated and analysed using Perl scripts and the R statistical package. A total of 1501 phenotypes were considered, for a per-SNP Bonferroni correction of $0.05 / 1501=3.3 \times 10^{-5}$

We then performed PheWAS for missense SNPs for each of the target genes from the mouse PheWAS that had minor allele frequencies $>1 \%$ and passed quality control filters of SNP call rate $>95 \%$ and sample call rate $>99 \%$ in unrelated samples. SNPs were found for ENPTD2 ( $r 334618694)$, COL6A5 ( $r s 1353613, r s 79867908, r s 12488457, r s 113396273, r s 35886424, r s 1453241$, $r s 1497312, r s 11917356, r s 76864445, r s 16827497, r s 16827168, r s 819085, r s 9883988$ 
and $r s 61744488), A H R$ ( $r s 2066853), A L A D(r s 1800435)$ and HDH3 (rs1043836). No SNPs were available for $\mathrm{FH} 1$.

All studies were approved by local Institutional Review Boards. Patients gave consent as part of the DNA biobanks at Group Health Cooperative, Marshfield Clinic, Mayo Clinic, Northwestern University; Vanderbilt uses an opt-out model as previously described and evaluated ${ }^{56,57}$.

C. elegans experiments. C. elegans were cultured at $20^{\circ} \mathrm{C}$ on nematode growth media agar plates seeded with bacteria. Strains were provided by the Caenorhabditis Genetics Center (University of Minnesota). The strains used were SJ4100 (zcIs13[hsp-6::GFP]), SJ4005 (zcIs4[hsp-4::GFP] and dvIs70 [hsp-16.2p::GFP + rol-6(su1006)]. RNAi constructs were isolated from the RNAi feeding library (GeneService) and experiments were carried out using standard feeding methods. The identity of each RNAi clone was verified by sequencing. RNAi treatment was started at embryonic stage. GFP was monitored in day 1 adults. Worms were immobilized with $6 \mathrm{mM}$ solution of tetramisole hydrochloride (Sigma) in M9 and imaged using Nikon DS-L2 fluorescent microscope.

\section{References}

1. Botstein, D. \& Risch, N. Discovering genotypes underlying human phenotypes: past successes for mendelian disease, future approaches for complex disease. Nat. Genet. 33, 228-237 (2003).

2. Cooper, G. M. \& Shendure, J. Needles in stacks of needles: finding diseasecausal variants in a wealth of genomic data. Nat. Rev. Genet. 12, 628-640 (2011).

3. Jelier, R., Semple, J. I., Garcia-Verdugo, R. \& Lehner, B. Predicting phenotypic variation in yeast from individual genome sequences. Nat. Genet. 43, 1270-1274 (2011)

4. Keane, T. M. et al. Mouse genomic variation and its effect on phenotypes and gene regulation. Nature 477, 289-294 (2011).

5. Nolan, P. M. et al. A systematic, genome-wide, phenotype-driven mutagenesis programme for gene function studies in the mouse. Nat. Genet. 25, 440-443 (2000)

6. Denny, J. C. et al. Systematic comparison of phenome-wide association study of electronic medical record data and genome-wide association study data. Nat. Biotechnol. 31, 1102-1110 (2013).

7. Denny, J. C. et al. PheWAS: demonstrating the feasibility of a phenome-wide scan to discover gene-disease associations. Bioinformatics. 26, 1205-1210 (2010).

8. Andreux, P. A. et al. Systems genetics of metabolism: the use of the BXD murine reference panel for multiscalar integration of traits. Cell 150, 1287-1299 (2012)

9. Chesler, E. J. et al. Complex trait analysis of gene expression uncovers polygenic and pleiotropic networks that modulate nervous system function. Nat. Genet. 37, 233-242 (2005)

10. $\mathrm{Wu}, \mathrm{Y}$. et al. Multilayered genetic and omics dissection of mitochondrial activity in a mouse reference population. Cell 158, 1415-1430 (2014).

11. Adzhubei, I. A. et al. A method and server for predicting damaging missense mutations. Nat. Methods. 7, 248-249 (2010).

12. Ng, P. C. \& Henikoff, S. SIFT: predicting amino acid changes that affect protein function. Nucleic Acids Res. 31, 3812-3814 (2003)

13. Aartsma-Rus, A., Janson, A. A. M., van Ommen, G. J. B. \& van Deutekom, J. C. T. Antisense-induced exon skipping for duplications in Duchenne muscular dystrophy. BMC. Med. Genet. 8, 43 (2007).

14. Ema, M. et al. Dioxin binding activities of polymorphic forms of mouse and human arylhydrocarbon receptors. J. Biol. Chem. 269, 27337-27343 (1994).

15. Guidry, P. A. \& Stroynowski, I. The murine family of gut-restricted class Ib MHC includes alternatively spliced isoforms of the proposed HLA-G homolog, 'blastocyst MHC'. J. Immunol. 175, 5248-5259 (2005).

16. Wang, X. et al. A promoter polymorphism in the Per3 gene is associated with alcohol and stress response. Transl. Psychiatry 2, 81-85 (2012).

17. Williams, E. G. et al. An evolutionarily conserved role for the aryl hydrocarbon receptor in the regulation of movement. PLoS Genet. 10, e1004673 (2014).

18. Tomlinson, I. P. et al. Germline mutations in FH predispose to dominantly inherited uterine fibroids, skin leiomyomata and papillary renal cell cancer. Nat. Genet. 30, 406-410 (2002).

19. Houtkooper, R. H. et al. Mitonuclear protein imbalance as a conserved longevity mechanism. Nature 497, 451-457 (2013).

20. Sena, L. A. et al. Mitochondria are required for antigen-specific T cell activation through reactive oxygen species signaling. Immunity 38, 225-236 (2013).

21. Gara, S. K. et al. Three novel collagen VI chains with high homology to the alpha3 chain. J. Biol. Chem. 283, 10658-10670 (2008).

22. Chang, C. et al. Ten nucleotide differences, five of which cause amino acid changes, are associated with the Ah receptor locus polymorphism of C57BL/6 and DBA/2 mice. Pharmacogenetics 3, 312-321 (1993).

23. Papadatos, G. A. et al. Slowed conduction and ventricular tachycardia after targeted disruption of the cardiac sodium channel gene Scn5a. Proc. Natl Acad. Sci. USA 99, 6210-6215 (2002).
24. Park, S. G. et al. The novel cytokine p43 stimulates dermal fibroblast proliferation and wound repair. Am. J. Pathol. 166, 387-398 (2005).

25. Siednienko, J. et al. Pellino3 targets the IRF7 pathway and facilitates autoregulation of TLR3- and viral-induced expression of type I interferons. Nat. Immunol. 13, 1055-1062 (2012).

26. Tsai, C. Y. et al. Hurp deficiency in mice leads to female infertility caused by an implantation defect. J. Biol. Chem. 283, 26302-26306 (2008).

27. Kumar, A. et al. Transgenic overexpression of cardiac actin in the mouse heart suggests coregulation of cardiac, skeletal and vascular actin expression. Transgenic Res. 13, 531-540 (2004).

28. Nowak, K. J. et al. Rescue of skeletal muscle alpha-actin-null mice by cardiac (fetal) alpha-actin. J. Cell Biol. 185, 903-915 (2009).

29. Ziebarth, J. D. et al. Treatment- and population-dependent activity patterns of behavioral and expression QTLs. PLoS ONE 7, e31805 (2012).

30. Runkel, E. D., Liu, S., Baumeister, R. \& Schulze, E. Surveillance-activated defenses block the ROS-induced mitochondrial unfolded protein response. PLoS Genet. 9, el003346 (2013).

31. Mouchiroud, L. et al. The $\mathrm{NAD}(+) /$ sirtuin pathway modulates longevity through activation of mitochondrial UPR and FOXO signaling. Cell 154, 430-441 (2013).

32. Zhao, Q. et al. A mitochondrial specific stress response in mammalian cells. EMBO J. 21, 4411-4419 (2002).

33. Durieux, J., Wolff, S. \& Dillin, A. The cell-non-autonomous nature of electron transport chain-mediated longevity. Cell 144, 79-91 (2011).

34. McGary, K. L. et al. Systematic discovery of nonobvious human disease models through orthologous phenotypes. Proc. Natl Acad. Sci. USA 107, 6544-6549 (2010).

35. Yamamoto, S. et al. A Drosophila genetic resource of mutants to study mechanisms underlying human genetic diseases. Cell 159, 200-214 (2014).

36. Huang, W. et al. Natural variation in genome architecture among 205 Drosophila melanogaster genetic reference panel lines. Genome Res. 24, 1193-1208 (2014).

37. Fu, J. et al. System-wide molecular evidence for phenotypic buffering in Arabidopsis. Nat. Genet. 41, 166-167 (2009).

38. Klassen, T. et al. Exome sequencing of ion channel genes reveals complex profiles confounding personal risk assessment in epilepsy. Cell 145, 1036-1048 (2011).

39. Manu et al. Canalization of gene expression in the Drosophila blastoderm by gap gene cross regulation. PLoS Biol. 7, e1000049 (2009).

40. Barbaric, I., Miller, G. \& Dear, T. N. Appearances can be deceiving: phenotypes of knockout mice. Brief. Funct. Genomic. Proteomic. 6, 91-103 (2007).

41. Hartman, J. L. t., Garvik, B. \& Hartwell, L. Principles for the buffering of genetic variation. Science 291, 1001-1004 (2001)

42. Li, H., Ruan, J. \& Durbin, R. Mapping short DNA sequencing reads and calling variants using mapping quality scores. Genome Res. 18, 1851-1858 (2008).

43. Ye, K., Schulz, M. H., Long, Q., Apweiler, R. \& Ning, Z. M. Pindel: a pattern growth approach to detect break points of large deletions and medium sized insertions from paired-end short reads. Bioinformatics 25, 2865-2871 (2009).

44. Chen, K. et al. BreakDancer: an algorithm for high-resolution mapping of genomic structural variation. Nat. Methods 6, 677-681 (2009).

45. Chen, K. et al. BreakDancer: an algorithm for high-resolution mapping of genomic structural variation. Nat. Methods 6, 677-U76 (2009).

46. Yoon, S., Xuan, Z., Makarov, V., Ye, K. \& Sebat, J. Sensitive and accurate detection of copy number variants using read depth of coverage. Genome Res. 19, 1586-1592 (2009).

47. Siepel, A. et al. Evolutionarily conserved elements in vertebrate, insect, worm, and yeast genomes. Genome Res. 15, 1034-1050 (2005).

48. Pollard, K. S., Hubisz, M. J., Rosenbloom, K. R. \& Siepel, A. Detection of nonneutral substitution rates on mammalian phylogenies. Genome Res. 20, 110-121 (2009).

49. Katoh, K. \& Toh, H. Recent developments in the MAFFT multiple sequence alignment program. Brief. Bioinform. 9, 286-298 (2008).

50. Lu, L., Airey, D. C. \& Williams, R. W. Complex trait analysis of the hippocampus: mapping and biometric analysis of two novel gene loci with specific effects on hippocampal structure in mice. J. Neurosci. 21, 3503-3514 (2001).

51. Chesler, E. J. et al. Genetic correlates of gene expression in recombinant inbred strains: a relational model system to explore neurobehavioral phenotypes. Neuroinformatics 1, 343-357 (2003).

52. Chesler, E. J., Lu, L., Wang, J., Williams, R. W. \& Manly, K. F. WebQTL: rapid exploratory analysis of gene expression and genetic networks for brain and behavior. Nat. Neurosci. 7, 485-486 (2004).

53. Storey, J. D. \& Tibshirani, R. Statistical significance for genomewide studies. Proc. Natl Acad. Sci. USA 100, 9440-9445 (2003).

54. Pritchard, J. K., Stephens, M. \& Donnelly, P. Inference of population structure using multilocus genotype data. Genetics 155, 945-959 (2000). 
55. Purcell, S. et al. PLINK: a tool set for whole-genome association and population-based linkage analyses. Am. J. Hum. Genet. 81, 559-575 (2007).

56. Roden, D. M. et al. Development of a large-scale de-identified DNA biobank to enable personalized medicine. Clin. Pharmacol. Ther. 84, 362-369 (2008).

57. Pulley, J., Clayton, E., Bernard, G. R., Roden, D. M. \& Masys, D. R. Principles of human subjects protections applied in an opt-out, de-identified biobank. Clin. Transl. Sci. 3, 42-48 (2010).

\section{Acknowledgements}

This work was supported by NIH grants R01AG043930, U01 AA016662, U01 AA013499 (R.W.W.), R01-LM010685, UL1 RR024975 and UL1 TR000445 (J.C.D.), the UTHSC Center for Integrative and Translational Genomics and the UT-Oak Ridge National Laboratory Governor Chair (R.W.W. and L.L.), the Gladstone Institutes (K.S.P. and J.A.C.), the EPFL, the Swiss Initiative for Systems Biology (51RTP0-151019 and 2013/ 153), SNSF (31003A-140780 and CSRII3-136201), the NIH grant R01 GM072285 (I.B.Z.), the Nestlé Chair in Energy Metabolism (J.A.) and the American Lebanese Syrian Associated Charities (J.P.).

\section{Author contributions}

R.W.W., L. L., X.W. and A.K.P. designed all experiments. X.W. and A.P. led the sequencing analysis. D.C.C., Z.L. and W.L.T. performed sample preparation and generated sequencing data using ABI SOLiD. Z.C. and S.F.N. generated sequencing data using illumina GA2. M.K.M., E.G.W. and K.M. provided bioinformatics support. E.G.W., V.J. and J.A. performed siRNA experiment. L.D.Q., Z.X., J.H. performed functional works on Col6a5. J.A.C. and K.S.P. performed functional analysis of noncoding SNPs. A.O.R., A.V.T., I.B.Z. performed functional analysis for coding SNP. X.W. and J.P. generated proteomics data and bioinformatics analysis. J.C.D., J.A. and
R.W.W preformed critical reading and contributed to the writing of the manuscript. X.W., A.K.P., M.K.M. and R.W.W. wrote the manuscript.

\section{Additional information}

Accession codes: The raw sequencing data has been submitted to the NCBI Sequence Read Archive under accession number SRA009489. Variant calls for both data sets are available via a mirror site of the UCSC genome browser (ucscbrowser.genenetwork.org). Additionally, 4.875 million SNPs and 0.67 million indels have been submitted to the dbSNP database (www.ncbi.nlm.nih.gov/SNP) using the submitter handle UTHSCSEQ.

Supplementary Information accompanies this paper at http://www.nature.com/ naturecommunications

Competing financial interests: The authors declare no competing financial interests.

Reprints and permission information is available online at http://npg.nature.com/ reprintsandpermissions/

How to cite this article: Wang, X. et al. Joint mouse-human phenome-wide association to test gene function and disease risk. Nat. Commun. 7:10464 doi: 10.1038/ncomms10464 (2016).

This work is licensed under a Creative Commons Attribution 4.0 International License. The images or other third party material in this article are included in the article's Creative Commons license, unless indicated otherwise in the credit line; if the material is not included under the Creative Commons license, users will need to obtain permission from the license holder to reproduce the material. To view a copy of this license, visit http://creativecommons.org/licenses/by/4.0/ 